PROCEEDINGS OF THE

AMERICAN MATHEMATICAL SOCIETY

Volume 136, Number 12, December 2008, Pages 4139-4142

S 0002-9939(08)09431-8

Article electronically published on June 17, 2008

\title{
A COUNTEREXAMPLE TO THE MAXIMALITY OF TORIC VARIETIES
}

\author{
VALERIE HOWER \\ (Communicated by Ted Chinburg)
}

\begin{abstract}
We present a counterexample to the conjecture of Bihan, Franz, McCrory, and van Hamel concerning the maximality of toric varieties. There exists a six dimensional projective toric variety $X$ with the sum of the $\mathbb{Z}_{2}$ Betti numbers of $X(\mathbb{R})$ strictly less than the sum of the $\mathbb{Z}_{2}$ Betti numbers of $X(\mathbb{C})$.
\end{abstract}

Let $X=X(\mathbb{C})$ be a complex algebraic variety defined by equations with real coefficients. Complex conjugation $\sigma: X \rightarrow X$ acts on $X$, and the fixed points $X^{\sigma}$ are the real points of $X$, denoted $X(\mathbb{R})$. It is of interest to try to understand the relationship between the topology of $X(\mathbb{C})$ and that of $X(\mathbb{R})$. If one looks at the $\mathbb{Z}_{2}$ Borel-Moore Betti numbers, the Smith-Thom inequality Deg, §1.1, states

$$
\sum_{i} b_{i}(X(\mathbb{R})) \leq \sum_{j} b_{j}(X(\mathbb{C})) .
$$

We say $X$ is maximal when equality is achieved.

Toric varieties are defined by equations with integral coefficients. See Fulton Ful] for background on toric varieties. Thus, if $X$ is a toric variety, we may consider $X(\mathbb{R})$ the real points of $X$. In $\mathrm{Bih}$, Bihan et al. define a spectral sequence $G_{p, q}^{r}$ that converges to the $\mathbb{Z}_{2}$ Borel-Moore homology of $X(\mathbb{R})$ when $X$ is a toric variety. We restrict ourselves to projective toric varieties, where the $\mathbb{Z}_{2}$ BorelMoore homology is isomorphic to the ordinary $\mathbb{Z}_{2}$ homology. We also use different notation and indexing than in $\mathrm{Bih}$. We write this spectral sequence as $\bar{E}_{p, q}^{r}$, where $G_{p, q}^{r}=\bar{E}_{p+q,-p}^{r}$. For each $p$ and $q$ we have

$$
\bar{E}_{p, q}^{1} \cong E_{p, q}^{2},
$$

where $E_{p, q}^{r}$ is the $\mathbb{Z}_{2}$ Leray spectral sequence for the moment map of $X(\mathbb{C})$. The spectral sequence $E_{p, q}^{r}$ converges to the ordinary $\mathbb{Z}_{2}$ homology of $X(\mathbb{C})$. Bihan et al. conjecture that every toric variety is maximal. Their conjecture is based on (1), numerous examples, and the fact that every nonsingular complete toric variety is maximal. In this paper, we present a counterexample to this conjecture. The author would like to thank Matthias Franz and Clint McCrory for ongoing discussions and emails.

Theorem. There exists a six dimensional projective toric variety which is not maximal.

Received by the editors May 4, 2007, and, in revised form, November 1, 2007.

2000 Mathematics Subject Classification. Primary 14M25, 14F45; Secondary 05B35.

(C)2008 American Mathematical Society 
To construct this toric variety, we will use a specific matroid. Matroids abstract the theory of dependence studied in linear algebra or graph theory. There are many equivalent definitions of a matroid; most are discussed in Oxl]. A definition useful for our purposes involves a convex polytope $P_{M}$. Let $E \subset\left(\begin{array}{c}{[n]} \\ k\end{array}\right)$ be a collection of $k$ element subsets of the ground set $[n]:=\{1,2,3, \ldots, n\}$. We define a convex polytope $P$ via

$$
P:=\operatorname{conv}\left\{e_{I}=\sum_{i \in I} e_{i} \mid I \in E\right\},
$$

where $e_{i}$ is the $i$ th standard basis vector for $\mathbb{R}^{n}$. If each edge of $P$ is parallel to $e_{i}-e_{j}$ for some $i, j$, then the set $E$ is the set of bases of a matroid $M$. In this case $P=P_{M}$ is the matroid polytope for the matroid $M$. Subsets of bases are independent sets, and the rank of a matroid is the cardinality of one of its bases.

We define a toric variety from a matroid as in Gel. Let $M$ be the rank 3 matroid $F_{7}$, the Fano plane. We remind the reader that $M$ is the projective plane over $\mathbb{F}_{2}$. The affine dependencies of $M$ are depicted below, and the bases of $M$ are the triangles in the diagram.

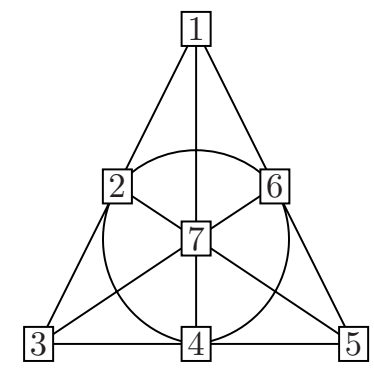

Next, consider the projective space $\mathbb{C P}^{27}$ whose coordinates are given by the bases of $M$

$$
\left\{y_{i j k} \mid i j k \in\left(\begin{array}{c}
{[7]} \\
3
\end{array}\right)-\{123,147,156,246,257,345,367\}\right\} .
$$

We define a linear action of the complex algebraic torus

$$
T:=\left(\mathbb{C}^{*}\right)^{7}=\left\{\left(t_{1}, t_{2}, t_{3}, t_{4}, t_{5}, t_{6}, t_{7}\right) \mid t_{i} \in \mathbb{C}^{*}\right\}
$$

on $\mathbb{C P}^{27}$ where the action on the coordinates is

$$
\left(t_{1}, t_{2}, t_{3}, t_{4}, t_{5}, t_{6}, t_{7}\right) \cdot y_{i j k}=t_{i} t_{j} t_{k} y_{i j k} .
$$

Note that the one-dimensional subtorus $\left\{(t, t, t, t, t, t, t) \mid t \in \mathbb{C}^{*}\right\}$ acts trivially. By restricting this action to the subtorus

$$
T^{\prime}:=\left\{\left(t_{1}, t_{2}, t_{3}, t_{4}, t_{5}, t_{6}, 1\right) \mid t_{i} \in \mathbb{C}^{*}\right\},
$$

we obtain an effective action of a six-dimensional algebraic torus on $\mathbb{C P}^{27}$. Let $X(\mathbb{C})$ be the closure of the torus orbit $T^{\prime} \cdot(1,1,1, \ldots, 1)$ in $\mathbb{C P}^{27}$. Then, $X$ is a sixdimensional projective toric variety. The moment polytope for $X$ is the projection of the matroid polytope $P_{M}$ onto the first six coordinates. We use torhom [Fra] to compute the ranks of the entries in $E_{p, q}^{2}=\bar{E}_{p, q}^{1}$ for $X$, which are listed in (2) , and 
the $\mathbb{Z}_{2}$ Betti numbers for $X(\mathbb{R})$. The $p$ th entry of the vector (3) is the rank of the $\mathbb{Z}_{2}$ vector space $H_{p}\left(X(\mathbb{R}), \mathbb{Z}_{2}\right)$.

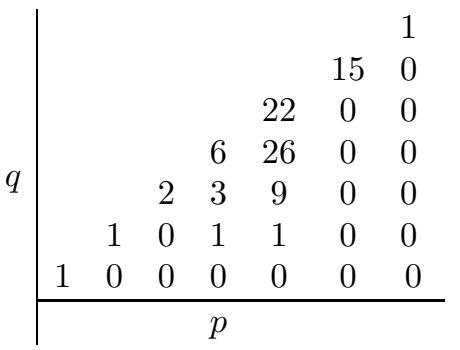

$$
\begin{aligned}
& {\left[\begin{array}{lllllll}
1 & 1 & 1 & 8 & 57 & 15 & 1
\end{array}\right]}
\end{aligned}
$$

As the total grading of the spectral sequence $\bar{E}_{p, q}^{r}$ is $p$, we see that the spectral sequence $\bar{E}_{p, q}^{r}$ does not collapse at $\bar{E}^{1}$. Moreover,

$$
\sum_{i=0}^{6} b_{i}(X(\mathbb{R}))=84 \text {. }
$$

The total grading for the spectral sequence $E_{p, q}^{r}$ is $p+q$. Thus, the only possible nonzero higher differential is $d_{4,1}^{2}: E_{4,1}^{2} \rightarrow E_{2,2}^{2}$. If $d_{4,1}^{2}=0$, then the spectral sequence collapses at $E^{2}$, the $\mathbb{Z}_{2}$ Betti numbers for $X(\mathbb{C})$, are

$$
\left[\begin{array}{lllllllllllll}
1 & 0 & 1 & 0 & 3 & 4 & 15 & 26 & 22 & 0 & 15 & 0 & 1
\end{array}\right]
$$

and

$$
\sum_{i=0}^{12} b_{i}(X(\mathbb{C}))=88
$$

If $d_{4,1}^{2} \neq 0$, then the $\mathbb{Z}_{2}$ Betti numbers for $X(\mathbb{C})$ are

$$
\left[\begin{array}{lllllllllllll}
1 & 0 & 1 & 0 & 2 & 3 & 15 & 26 & 22 & 0 & 15 & 0 & 1
\end{array}\right]
$$

and

In either case, we obtain

$$
\sum_{i=0}^{12} b_{i}(X(\mathbb{C}))=86
$$

$$
\sum_{i=0}^{6} b_{i}(X(\mathbb{R}))<\sum_{i=0}^{12} b_{i}(X(\mathbb{C})),
$$

and hence $X$ is not maximal.

We do not know why $X$ fails to be maximal. Nonetheless, this example does have some remarkable properties. For instance, the Fano plane is a matroid which is not realizable over any field $\mathbb{F}$ unless char $\mathbb{F}=2$. Moreover, the polytope $P_{M}$ with $f$-vector $(28,126,245,238,112,21)$ is highly symmetric; the symmetry group of $P_{M}$ is $\mathrm{PGL}_{3}\left(\mathbb{F}_{2}\right)$. However at the current time, we are unable to connect these unique properties of $M$ and $P_{M}$ to the topology of $X$.

Other than slightly larger examples which contain $X$ as the closure of a torus orbit, no other counterexamples are known. Hence, we have no intuition as to what features might distinguish maximal from nonmaximal toric varieties. As mentioned 
above, all nonsingular complete toric varieties are maximal, and there are certainly classes of singular toric varieties which are maximal. For instance, in $\mathrm{How}$ we show that toric varieties associated to Fano polyhedra (defined in Bat]) are maximal. This counterexample reveals that the relationship between the topology of the real and complex points of a toric variety is not as straightforward as one might hope.

In the future, we would like to see a description, perhaps combinatorial, of the class of maximal toric varieties.

\section{REFERENCES}

[Bat] V. Batyrev, Dual polyhedra and mirror symmetry for Calabi-Yau hypersurfaces in toric varieties, J. Algebraic Geom. 3 (1994), 493-535. MR.1269718 (95c:14046)

[Bih] F. Bihan, M. Franz, C. McCrory, J. van Hamel, Is every toric variety an M-variety?, Manuscripta Math. 120 (2006), 217-232. MR2234250

[Deg] A. Degtyarev, I. Itenberg, V. Kharlamov, Real Enriques Surfaces, Springer-Verlag, New York, 2000. MR1795406 (2001k:14100)

[Fra] M. Franz, Maple package torhom, version 1.3.0, September 13, 2004, Available at http://www-fourier.ujf-grenoble.fr/ franz/maple/torhom.html

[Ful] W. Fulton, Introduction to Toric Varieties, Princeton University Press, Princeton, NJ, 1993. MR 1234037 (94g:14028)

[Gel] I.M. Gelfand, M. Goresky, R.D. MacPherson, V.V. Serganova, Combinatorial geometries, convex polyhedra, and Schubert cells, Adv. Math. 63 (1987), 301-316. MR877789 (88f:14045)

[How] V. Hower, Hodge spaces of real toric varieties, Collect. Math. 59 (2008), 215-237.

[Oxl] J.G. Oxley, Matroid Theory, Oxford University Press, New York, 1992. MR1207587 (94d:05033)

Department of Mathematics, Georgia Institute of Technology, Atlanta, Georgia 30332

E-mail address: vhower@math.gatech.edu 